V. Krasnoholovets, Dark matter as seen from the physical point of view, Astrophysics and Space Science 335, No. 2, 619-627 (2011).

\title{
Dark matter as seen from the physical point of view
}

\author{
Volodymyr Krasnoholovets \\ Indra Scientific SA, Square du Solbosch 26, Brussels B-1050, Belgium \\ e-mail: v_kras@yahoo.com
}

7 May 2011

\begin{abstract}
It is shown that the Newton's law of universal gravitation can be derived from first submicroscopic principles inherent in the very nature of real space that is constituted as a tessel-lattice of primary topological balls. The submicroscopic concept determines the notion of mass in the tessel-lattice and introduces excitations of space, which appear at the motion of particles (mass particles are determined as local deformations of the tessel-lattice). These excitations are associated with carriers of the field of inertia. In the universe the gravitation is induced by standing inerton waves of mass objects, which oscillate around the objects with the speed of light. An overlapping of these standing inerton waves generates an elastic interaction between masses bringing them to a formation of clusters in which masses are characterised by both the Newtonian and elastic interaction. It is this elastic interaction that cancels the necessity of introduction of mystical dark matter. At the same time, inertons, carriers of inert properties of objects, can be treated as an analogous of hypothetic weakly interacting massive particles (WIMP) or axions, which some astronomers try to associate with dark matter particles.
\end{abstract}

Key words: dark matter, clusters of galaxies, gravitation, space, tessel-lattice, inertons

PACS: 04.20.Cv; 04.40.-b; 05.50.+q; 95.35.+d; 98.20.-d; 98.65.-r

\section{Introduction}

McGauph [1] has recently reported results of testing the baryonic Tully-Fisher relation (BTFR) for gas rich galaxies, i.e. for a class of galaxies where stars do not dominate the baryonic mass budget. At the testing both axes of the BTFR have been measured independently of the available theories. McGauph has demonstrated that his data coincide exactly with those predicted by Milgrom's hypothesis [2, 3]. Some more theoretical preference to the Milgrom's modified Newtonian dynamics (MOND) is given in a review article [4]. 
The starting point [2, 3] was a departure from Newtonian dynamics. Namely, the Newton force $\mathbf{F}=m \mathbf{a}$ was substituted for a significantly less force $\mathbf{F}=m \cdot(\mathbf{a}-\Delta \mathbf{a})$, which assumed to be active at a scale of small centripetal accelerations of stars in some galaxies. The modified regime is switched in at $a<<a_{0}$ where $a_{0}=$ $(1.21 \pm 0.24) \times 10^{-10} \mathrm{~ms}^{-2}$ is the value of the threshould acceleration estimated in paper [5]. It is a matter of fact that at such small $a$ the rotation curves of disc galaxies behave as flat: stars' orbital velocities $V \sim$ const, although in the classical case of Newton dynamics the curves have to follow a Keplerian law, $V \sim 1 / \sqrt{r}$.

Milgrom's approach allowed one to introduce a relation between a total mass $M$ of the galaxy studied and its rotation velocity [2, 5]

$$
V^{4}=G M a_{0}
$$

and it is this relationship that has recently been verified [1].

Moreover, McGuaph [1] further noted that MOND (i) predicted in advance that galaxies of both high and low surface brightness would fall on the same BTFR, though this contradicts to the expectation of purely Newtonian gravity; (ii) prescribed the mass-to-light ratios that agreed the stellar population synthesis models; (iii) provided the only successful a priori prediction of the first-to-second peak amplitude ratio of the acoustic peaks of the cosmic background radiation. McGuaph then reasonably concludes: "It is rare for a non-canonical theory to have so many predictive successes".

Notwithstanding this, [1 indicates also major shortcomings of MOND when applying to describe rich clusters of galaxies [6] and the bullet cluster [7: the discrepancy of the clusters mass is a factor of two, which means that MOND suffers of some kind of dark mass too (though in the case of the Lambda-Cold Dark Matter model the discrepancy of mass reaches $99 \%$ ).

Thus, to account for all the experimental results, MOND requires some dark matter particles. These dark matter particles should behave themselves as "standard cold dark" matter on large scales and at the same time have to interact with conventional matter resulting in MOND phenomenology for disc galaxies.

In the present paper those missing dark matter particles are introduced starting from first submicroscopic principles.

\section{Preliminaries}

\subsection{Mathematical background}

Some researchers mention the incompleteness of general relativity and the difficulties associated with its physical interpretation. The others talk about conceptual difficulties of quantum mechanics. And everyone understands that these two theories are quite different and unification is possible only with substantial changes in both concepts. Conciliation is feasible only on a common physical base, which is beyond the formalism of both quantum mechanics and general relativity. This would be rather a kind of the double solution theory over which de Broglie [8] was working since the beginning of 1950-s.

So, what would be the starting point, a basis for a more universal physical theory? The theory of everything tries to describe the whole physical world starting 
from the first principles of quantum theory. However, what about a theory of something? It is this theory that is able to clarify the major primary notions of physics, namely: space, mass, charge, particle, field, etc.

The theory of something [9, 10, 11, 12] starts as a pure mathematical theory from set theory, topology and fractal geometry generating a common study of such fundamental notions, as measure, distance, space dimensions and the founding element (the empty set $\varnothing$ ). The axiom of the existence of the empty set, added with the axiom of availability, in turn provide existence to a lattice $\mathcal{L}(\varnothing)$ of empty set, which constitutes a discrete fractal space. The set of parts of $\varnothing$ contains parts equipotent to sets of integers, of rational and of real numbers, and owns the power of continuum. These spaces provide collections of discrete manifolds whose interior is endowed with the power of continuum. Any of intersections of subspaces provide a $(\mathrm{D}<4)$-space in which closed members get the status of both observable objects and perceiving objects. This stands for observability, which is a condition for a space to be in some sort observable, that is physical-like.

Therefore, this mathematical lattice of empty set cells to be able to account for a primary degenerate substrate [9, 12]. Space-time is represented by ordered sequences of topologically closed Poincaré sections of this primary space. These mappings are constrained to provide homeomorphic structures serving as frames of reference in order to account for the successive positions of any objects present in the system. Discrete properties of the lattice, called a tessel-lattice, allow the prediction of scales at which microscopic to cosmic structures should occur.

Deformations of primary cells by exchange of empty set cells allow a cell to be mapped into an image cell in the next section as far as mapped cells remain homeomorphic. However, if a deformation involves a fractal transformation to objects, there occurs a change in the dimension of the cell and the homeomorphism is not conserved. Then the fractal kernel of such deformed cell of the tessel-lattice can stand for a "particle" and the reduction of its volume (together with an increase of its area) is compensated by morphic changes of a finite number of surrounding cells.

It is obvious that in the tessel-lattice a moving particle-like deformation has to interact with the surrounding cells involving a fractal decomposition process: the particulate cell exchanges its original deformation with the surrounding cells, which will result in the appearance of a kind of a cloud of deformed cells enclosing the particulate cell.

\subsection{The emergence of quantum physics in the tessel- lattice}

A volumetric fractal deformation of a cell of the tessel-lattice can be associated with the physical notion of mass, $m=C \mathcal{V}_{\text {deg. cell }} / \mathcal{V}_{\text {deform.cell }}$, where $C$ is a dimension constant and $\mathcal{V}_{\text {deg.cell }}$ stands for the volume of an original degenerate cell and $\mathcal{V}_{\text {deform.cell }}$ for the volume of the deformed cell. In physics a resistance to the motion is called inertia. That is why excitations of the tessel-lattice produced by a moving particulate cell were called inertons.

If we consider kinetics of the motion of such complex object - a particle surrounded with a cloud of inertons, - we derive [13, 14] relationships for a particle 
suggested by Louis de Broglie in 1924

$$
E=h \nu, \quad \lambda=h /(m v)
$$

Since relationships (2) have been obtained for a particle moving in the tessellattice, the sense of parameters in (2) becomes very clear: $E$ is the total energy of the particle, $m v$ is its momentum, $\lambda$ is the spatial period in which the particle emits inertons and then absorb them back, $\nu$ is the frequency of collisions of the particle with its cloud of inertons. Because of the periodicity, the moving particle periodically emits and absorbs its inertons, it is characterised by an increase in its action $\Delta S$; since this is a free motion through the degenerate tessel-lattice, this increment $\Delta S$ is associated with the Planck constant $h$.

De Broglie's relationships (2) allow one to obtain the Schrödinger wave equation [15]. But the relationships (2) obtained in our case signify that the Schrödinger wave $\psi$-function gains a real physical sense: $\psi$ represents the particle's field of inertia whose carriers are inertons and they carry mass and fractal properties of the particle [16]. The cloud of inertons occupies the section $\lambda$ along the particle's path and spreads to a distance of $\Lambda=\lambda c / v$ in transversal directions, where $c$ is the velocity of light for inertons in the particle's cloud (though a free inerton possesses a large velocity [17]).

An inerton field complete rejects the action at-a-distance from the realm of quantum physics and introduces determinism at every stage of evolution of the system studied [16]. This new submicroscopic concept could properly resolve quite a number of difficulties available in quantum mechanics. Moreover, the concept was successfully applied for the description of many experiments. Most interesting applications, which are in line with the subject of the present paper, are associated with a variation in mass of entities in condensed media (owing to the overlapping of inerton clouds of entities) [18] and the dense inerton field gathering of about $10^{10}$ electrons in a log-living cluster (due to the absorption of inertons knocked out by a laser beam from a crystal) [19.

The tessel-lattice is the source for an electric and magnetic charge, photon and electromagnetic field and these make it possible to derive the Maxwell equations [16].

\subsection{Gravitation in the tessel-lattice}

At last, the concept allows the derivation of the Newton's law of universal gravitation [20, 16], which is behind the formalism of general relativity. In the case of a canonical particle, the gravitation emerges when the tessel-lattice responses to inertons emitted by the moving particle, namely, the tessel-lattice elastically pushes them back to the particle and the latter re-absorbs them. This means the motion of a particle occurs with the periodical decay of its mass: the mass is gradually transformed into a tension of the tessel-lattice. The appropriate whole Lagrangian has the form [20]

$$
\begin{aligned}
L= & -m_{0} c^{2}\{\text { submicroscopic mechanics } \\
& \left.+\left(\frac{T^{2}}{2 m_{0}^{2}} \dot{m}^{2}+\frac{T^{2}}{2 \Lambda^{2}} \dot{\boldsymbol{\xi}}^{2}-\frac{T}{m_{0}} \dot{m} \nabla \boldsymbol{\xi}\right)\right\}^{1 / 2} .
\end{aligned}
$$


Here, $m_{0}$ is the rest mass of the particle, $\Lambda$ is the amplitude of the particle's inerton cloud, $T$ is the period of collisions of the particle and its inerton cloud; $m=$ $m(\boldsymbol{r}, t)$ is the current mass of the \{particle + inerton cloud $\}$-system; $\boldsymbol{\xi}=\boldsymbol{\xi}(\boldsymbol{r}, t)$ is the current value of the rugosity of the tessel-lattice in the range covered by the system. Geometrically the rugosity $\boldsymbol{\xi}$ depicts the state in which the tessel-lattice cells covered by the inerton cloud are shifted a little bit from their equilibrium positions, which induces a local tension in the tessel-lattice; the tension of the particulate cell may be associated with an increase in size.

The system studied features the radial symmetry; then variables $m$ and $\boldsymbol{\xi}$ are functions of only the distance $r$ from the particle and the proper time $t$ of the $\{$ particle + inerton cloud $\}$-system. In this case we preserve only radial components in the both variables, which enables us to use the spherical coordinates. The EulerLagrange equations of motions for $m$ and the radial component of $\boldsymbol{\xi}$ result in [20]

$$
\begin{aligned}
& \frac{\partial^{2} m}{\partial t^{2}}-\left(\frac{c^{2}}{r}\right) \frac{\partial^{2}(r m)}{\partial r^{2}}=0, \\
& \frac{\partial^{2} \xi}{\partial t^{2}}-\left(\frac{\Lambda^{2}}{m_{0} T}\right) \frac{\partial}{\partial r}\left(\frac{\partial m}{\partial t}\right)=0
\end{aligned}
$$

where the Laplace operator is presented in the spherical coordinates as $\Delta m=$ $\frac{1}{r} \frac{\partial^{2}}{\partial r^{2}}(r m)$.

The radial symmetry allows the solutions to equations (44) and (5) in the form of standing spherical waves, which exhibit the dependence $1 / r$,

$$
\begin{gathered}
m(r, t)=C_{1} \frac{m_{0}}{r} \cos \left(\frac{\pi r}{2 \Lambda}\right)\left|\cos \left(\frac{\pi t}{2 T}\right)\right| \\
\xi(r, t)=C_{2} \frac{\xi_{0}}{r} \sin \left(\frac{\pi r}{2 \Lambda}\right) \cdot(-1)^{\left[\frac{t}{T}\right]} \cdot\left|\sin \left(\frac{\pi t}{2 T}\right)\right| .
\end{gathered}
$$

The dimensionality of integration constants $C_{1,2}$ corresponds to length and can be put here $C_{1}=l_{\text {Planck }} \approx 10^{-35} \mathrm{~m}$ and $C_{2}=\Lambda$.

An object, which consists of many particles (a solid, a planet, or a star), experiences local vibrations of its entities (atoms, ions, particles), as is the case with entities in the crystal lattice. Entities vibrate in the neighborhood of their equilibrium positions and/or move to new positions. These movements produce inerton clouds around the appropriate particles. Inerton clouds overlap forming a total inerton cloud of the object [20, 16]. The spectrum of inertons is similar to the spectrum of phonons in a solid (a body of phonons is filled with inerton carriers) [18]. For instance, if we have a solid sphere with a radius $R_{\mathrm{sph}}$, which consists of $N_{\mathrm{sph}}$ atoms, the spectrum of acoustic waves will include $N_{\mathrm{sph}} / 2$ wave harmonics with wavelengths $\lambda_{n}=2 b n$ where $b$ is the lattice constant and $n=1,2,3, \ldots, N_{\mathrm{sph}} / 2$.

At the same time, overlapping inerton clouds, which accompany vibrating atoms, produce their own spectrum of inerton wavelengths

$$
\Lambda_{n}=\lambda_{n} c / v_{\text {sound }} \text {. }
$$

This means that the Lagrangian (3) and the solutions (6) and (7) obtained for a moving particle are proper also for a macroscopic object at rest. Indeed, an object with a mass $m$ is characterised by the inner motion of its entities, whose vibrations induce oscillations of an inerton field both inside and outside of the object. 
For instance, for a small solid sphere with a size $1 \mathrm{~cm}^{3}$ and the quantity of atoms $N_{\text {sph }} \sim 10^{22}$ we get from relation (8) that the longest standing inerton wave can spread up to a distance $\Lambda \sim 10^{17} \mathrm{~m} \approx 10.5$ light years. Behind this radius any information about the solid sphere is absent.

Longest standing inerton waves of a macroscopic body with a radius $R$ and a mass $m$ induce the deformation potential (6) in the surrounding space, i.e. these waves contract cells of the tessel-lattice as the rule (6) prescribes. In the region of space $R \leq r<<\Lambda$ the time-averaged distribution of the mass of the standing inerton wave becomes

$$
m(r, t)=l_{\text {Planck }} m_{0} / r .
$$

Multiplying both hand sides of expression (9) by a factor of $-G / l_{\text {Planck }}$ we obtain a conventional Newtonian potential

$$
U=-G m_{0} / r
$$

that describes the gravitational attraction of a test mass to the object with the mass $m_{0}$.

This result shows that the gravitational mass of the object is complete accumulated in its standing inerton wave.

This theory of the Newtonian potential formation has further been developed in works [21, 22]. In particular, it has been argued the necessity of the tangential inerton interaction between masses, which gives rise to a correction to the Newton's law of gravitation [21]

$$
U=-G \frac{m_{0} m_{1}}{r}\left(1+\frac{\dot{r}_{\tan }^{2}}{c^{2}}\right)
$$

where $\dot{r}_{\text {tan }}$ is the tangential velocity of a test body with the mass $m_{1}$ i.e. the body's orbital velocity. Corrected Newton's law of gravitation (11) was further used to study the anomalous precession of the Mercury's perihelion, the bending of light and the red shift of spectral lines [21]. The submicroscopic concept made it possible to derive exactly the same equations for the descriptions of those three phenomena, which were produced by general relativity. Moreover, the submicroscopic concept has enabled one to clarify a nature of changes in space associated with the so-called gravitational time delay effect (the Shapiro time delay effect) [22]. It is important to note that the velocity of inertons in standing inerton waves may exceed the speed of light, because their velocity includes also the velocity of the object that irradiates

these standing waves (i.e., $v_{\text {stand. inertons }}=\sqrt{c^{2}+v_{\text {object }}^{2}}$, see the expression (11) and Ref. [21]).

\section{Interacting stars}

\subsection{Statistical mechanical approach}

Krasnoholovets and Lev [23] developed a method of statistical physics for the description of systems of interacting particles taking into account a spatial nonhomogeneous distribution of particles, i.e. cluster formation. In particular, we considered gravitating masses with the Hubble expansion. 
Identical gravitating masses, i.e. stars, were characterised by an effective attraction potential energy $u_{12}^{\text {attr. }}=G m^{2} /\left(r_{1}-r_{2}\right)$ and the effective repulsion energy $u_{12}^{\text {repul. }}=\frac{1}{2} m H_{0}^{2} \cdot\left(r_{1}-r_{2}\right)^{2}$. We used the fact that an additional kinetic energy $E_{12}=\frac{1}{2} m \cdot\left(v_{1}-v_{2}\right)^{2}$ of masses is associated with the Hubble expansion. The relative velocity $v_{1}-v_{2}$ of masses separated in space correlates with the relative distance between the masses, because $v_{1}-v_{2}=H_{0} \cdot\left(r_{1}-r_{2}\right)$, where $H_{0}$ is the Hubble constant.

We obtained the following solution for a number of stars gathered in a cluster 23

$$
\aleph \approx \frac{120 \pi}{33} \frac{G \rho}{H_{0}^{2}}
$$

where $\rho$ is the density of stars. As seen from expression (12), just value of $\rho$ is critical for the formation of a cluster, i.e. when $\aleph>>1$. Putting $H_{0}=1.6 \times 10^{18} \mathrm{~s}^{-1}$, we may state that the inequality $\aleph>>1$ holds when the density of stars $\rho>>10^{-25}$ $\mathrm{kg} \mathrm{m}^{-3} \approx \mathrm{M}_{\odot} /(11 \mathrm{kpc})^{3}$.

Now let us come back to the Newtonian gravitational potential (10), which is formed by standing inerton waves of an object with a mass $m$. In the case of the sun, the number of its particles is above $10^{50}$. Then at the worse conditions for relation (8), $\lambda_{1} \sim 10^{-15} \mathrm{~m}$ and $c / v_{\text {sound }} \sim 1$, we get $\lambda_{N / 2} \sim 10^{35} \mathrm{~m}$ and may crude estimate a boundary to which the sun's inertons can spread: $\Lambda_{N / 2} \sim 10^{35} \mathrm{~m}$, which exceeds the observed radius of the universe. This means that we may neglect the rugosity/tension (7) of the tessel-lattice at an examination of a system of stars, which are disposed in the same galaxy.

However, we cannot disregard an overlapping of local deformations of the tessellattice, induced by standing inerton waves of a system of stars. In fact, a "breathing" of a star, i.e. radial oscillations of its inerton clouds, which occur with the speed of light $c$, results in mutual overlapping of inerton clouds of stars. Therefore, these standing inerton waves induce the Newton's potential of gravitation (10) and, in addition, owing to the mutual scattering of counterpropagating waves of nearest stars they introduce the elasticity in interstellar space. This means that the quasistationary gravitational law (11) should be supplemented by an additional elastic energy created by the mutual overlapping of inerton clouds of all stars of the system studied. Note in such a way a unification of molecules takes place in gases, liquids and solids [18.

Thus, a correct expression for the energy of interacting stars should include four terms: (i) the gravitational potential interaction (11) between two masses $m_{i}$ and $m_{j}$; (ii) the gravitational interaction of a mass $m_{i}$ with the total mass $M$ of the system of stars; (iii) an elastic interaction between masses $m_{i}$ and $m_{j}$; (iv) the interaction between masses $m_{i}$ and $m_{j}$ associated with the Hubble expansion (see above).

Basing on the results [23], we may assume that all stars in the system studied are distributed by nodes of a lattice (some nodes are filled and some not). Then if a system of interacting particles possesses attraction and repulsion pair potentials, statistical mechanics prescribes 23 that in such a system all particles become distributed by $K$ identical clusters. The action for each cluster looks as follows

$$
S \approx(\alpha-\beta) \cdot \aleph^{2}
$$


where $\aleph$ is the number of particles in the cluster and $\alpha$ and $\beta$ are functions associated with the particle interactions:

$$
\begin{aligned}
& \alpha=\frac{1}{\wp k_{\mathrm{B}} \Theta} \int d \boldsymbol{r} u^{\text {elast. repul. }}(\boldsymbol{r}), \\
& \beta=\frac{1}{\wp k_{\mathrm{B}} \Theta} \int d \boldsymbol{r} u^{\text {attract. }}(\boldsymbol{r})
\end{aligned}
$$

where $\wp$ is the effective volume of a particle and the integration is running over the volume of the whole cluster; $k_{\mathrm{B}} \Theta$ is the thermal energy of the environmental thermostat $\left(k_{\mathrm{B}}\right.$ is the Boltzmann constant and $\Theta$ the absolute temperature). Absolute values of the pair potential interactions are

$$
\begin{aligned}
& u^{\text {elast. repuls. }}(g x)=\frac{1}{2} m \omega^{2} \cdot(g x)^{2}+\frac{1}{2} m H_{0}^{2} \cdot(g x)^{2}, \\
& u^{\text {attract. }}(g x)=\frac{G M m}{R}+\frac{G m^{2}}{g x}
\end{aligned}
$$

where $m$ is the mass of a star; $g$ is the lattice constant (a distance between neighbour stars in the model lattice); $x$ is the dimensionless distance defined through a relation $r=g x ; \omega$ is the radial frequency of oscillation of the mass $m$ near its equilibrium position; $M$ is the mass of the whole system of stars, which occupies space up to the effective radius $R$, and hence in expression (16) the first term can be considered as a middle-field potential energy.

\section{$3.2 \quad 3-\mathrm{D}$ clusters}

For a spherical cluster the integrals in expression (14) can be rewritten via the number $\aleph$ of particles in the cluster 23.

$$
\frac{1}{\wp} \int d \boldsymbol{r}=\frac{1}{(4 \pi / 3) g^{3}} 4 \pi \int_{g}^{R} r^{2} d r=\frac{R^{3}-g^{3}}{g^{3}}=\aleph .
$$

Substituting expressions (15) and (16) into the integrals (14) we get

$$
\begin{aligned}
\alpha & =\frac{3}{k_{\mathrm{B}} \Theta} \int_{1}^{\aleph^{1 / 3}} d x x^{2} u^{\text {elast. repuls. }}(g x) \\
& =\frac{3}{10 k_{\mathrm{B}} \Theta} m g^{2} \cdot\left(\omega^{2}+H_{0}^{2}\right) \aleph^{5 / 3}, \\
\beta & =\frac{3}{k_{\mathrm{B}} \Theta} \int_{1}^{\aleph^{1 / 3}} d x x^{2} u^{\text {attract. }}(g x) \\
& =\frac{3}{k_{\mathrm{B}} \Theta}\left(\frac{G M m}{3 R} \aleph+\frac{G m^{2}}{2 g} \aleph^{2 / 3}\right) .
\end{aligned}
$$

Then the action (13) becomes

$$
\begin{aligned}
S=\frac{1}{k_{\mathrm{B}} \Theta}\{ & \frac{3}{10} m \cdot\left(\omega^{2}+H_{0}^{2}\right) g^{2} \aleph^{11 / 3} \\
& \left.-\frac{G M m}{R} \aleph^{3}-\frac{3 G m^{2}}{2 g} \aleph^{8 / 3}\right\} .
\end{aligned}
$$

Taking into account the fact that galaxies and clusters consist at least of a few thousand stars and knowing typical values for $M, m, R$ and $g$, we may conclude 
that in the first approximation the last term in expression (20) can be neglected. Besides, it is reasonable to assume that the Hubble energy, which affects a star, is smaller than the elastic energy that retaining the star in a cluster. Therefore, the contribution on the side of $H_{0}$ may be considered as negligible. The simplified action (20) can be investigated for the extremum: $\partial S / \partial \aleph=0$. The solution to this equation is

$$
\aleph=\left(\frac{30}{11} \frac{G M}{R g^{2} \omega^{2}}\right)^{3 / 2} .
$$

\section{$3.3 \quad 2-\mathrm{D}$ clusters}

In the case of quasi-flat clusters the expression (17) changes to

$$
\frac{1}{A} \int d \boldsymbol{r}=\frac{1}{\pi g^{2}} 2 \pi \int_{g}^{R} r d r=\frac{R^{2}-g^{2}}{g^{2}}=\aleph
$$

where $A=\pi g^{2}$ is the area occupied by one particle in a cluster. Then retaining highest order terms in equation (14) written for the flat case, we obtain for the functions $\alpha$ and $\beta$ :

$$
\begin{aligned}
& \alpha=\frac{2}{k_{\mathrm{B}} \Theta} \int_{1}^{\aleph^{1 / 2}} d x x u^{\text {elast. repuls. }}(g x)=\frac{1}{2} \frac{m \omega^{2} g^{2}}{k_{\mathrm{B}} \Theta} \aleph^{2}, \\
& \beta=\frac{2}{k_{\mathrm{B}} \Theta} \int_{1}^{\aleph^{1 / 2}} d x x u^{\text {attract. }}(g x)=\frac{G M m}{R k_{\mathrm{B}} \Theta} .
\end{aligned}
$$

Having these functions, we construct the action (13) as follows

$$
S=\frac{1}{k_{\mathrm{B}} \Theta}\left\{\frac{1}{2} m \omega^{2} g^{2} \aleph^{4}-\frac{G M m}{R} \aleph^{3}\right\} .
$$

The extremum is achieved at the solution of the equation $\partial S / \partial \aleph=0$, which results in the solution

$$
\aleph=\frac{3}{2} \frac{G M}{R \omega^{2} g^{2}} .
$$

\subsection{1-D clusters}

In the case of quasi-linear clusters formulas are maximally simplified. Indeed, $\aleph=$ $R / g$ and the functions $\alpha$ and $\beta$ (14) become

$$
\begin{aligned}
& \alpha=\frac{1}{k_{\mathrm{B}} \Theta} \int_{1}^{\aleph} d x u^{\text {elast. repuls. }}(g x)=\frac{1}{3} \frac{m \omega^{2} g^{2}}{k_{\mathrm{B}} \Theta} \aleph^{3}, \\
& \beta=\frac{1}{k_{\mathrm{B}} \Theta} \int_{1}^{\aleph} d x u^{\text {attract. }}(g x)=\frac{G M m}{R k_{\mathrm{B}} \Theta} \aleph .
\end{aligned}
$$

Having functions (27) and (28), we obtain the action (13) as follows

$$
S=\frac{1}{k_{\mathrm{B}} \Theta}\left\{\frac{1}{3} m \omega^{2} g^{2} \aleph^{5}-\frac{G M m}{R} \aleph^{3}\right\}
$$

The solution of the equation $\partial S / \partial \aleph=0$ results in

$$
\aleph=\left(\frac{9}{5} \frac{G M}{R \omega^{2} g^{2}}\right)^{1 / 2} .
$$

Let us analyse the obtained solutions (21), (26) and (30). 


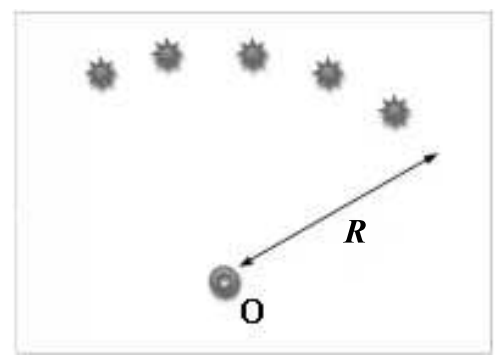

Figure 1: Arc formed by stars in the neighbour of the centroid of a galaxy, which is a typical quasi-1-D cluster.

\section{Discussion}

The solutions, which exhibit the distribution of particles by clusters with $\aleph$ particles per cluster, can be applied for the description of disc galaxies (expression (26) ) and star clusters (expression (21)). In fact, the phenomenon of cluster formation is well known in condensed matter physics, which occurs with the presence of an outside field. For example, in the presence of a thermal gradient the so-called RayleighBénard cells (identical cylindrical or hexagonal structures) appear in a layer of a primary uniform viscous fluid, electrons assemble in clusters (about $10^{8}$ electrons per cluster) on the surface of liquid helium and, at last, we [19] could generate a long-living clusters of electrons in which about $10^{10}$ electrons were gathering in one droplet where they were hold by an inerton field.

Arcs and arclets of stars have been observed and intensively investigated in rich clusters of galaxies [24]. 1-D cluster solution (30) exactly satisfies an arc of stars (Fig. 1). Indeed, $\aleph>>1$ is reached in a wide range of parameters. For example, putting $M \sim 10^{13} \mathrm{M}_{\odot}, R \sim 100 \mathrm{kpc}, g \sim 1 \mathrm{pc}$ and $\omega \sim 10^{-14} \mathrm{~s}^{-1}$, we get from (30): $\aleph \sim 10^{5}$ stars in an arc. Less values of $M$ will give less value of stars involved in an arc. It is interesting that in principle a ring distribution of stars is also quite possible.

For a disc galaxy we may apply the 2-D cluster solution (26), which means that all the stars of the disc galaxy are distributed by plane clusters with the appropriate number $\aleph$ of stars. Let us estimate the value of $\aleph$. In particular, for a disc gas galaxy we may choose some typical values of the mass, the radius and the distance between stars in the galaxy: $M=10^{8} \mathrm{M}_{\odot}[1], R=10 \mathrm{kpc}, g=1 \mathrm{pc}$. So, we have only one fit parameter, the frequency $\omega$ of oscillations of a star near its equilibrium position in the cluster. This parameter can be estimated from a relation that restrains a star in the cluster. Figure 1 depicts: star 1 experiences the centripetal acceleration $\boldsymbol{a}$ to the centre $\mathrm{O}$ of the galaxy; at the same time neighbour stars strongly keep it by means of the elastic energy $\frac{1}{2} m \omega^{2} \boldsymbol{r}^{2}$ (Fig. 2). This means that the equality of two accelerations is hold:

$$
G M / R^{2}-\omega^{2} g=0 .
$$

From equation (31) we get 


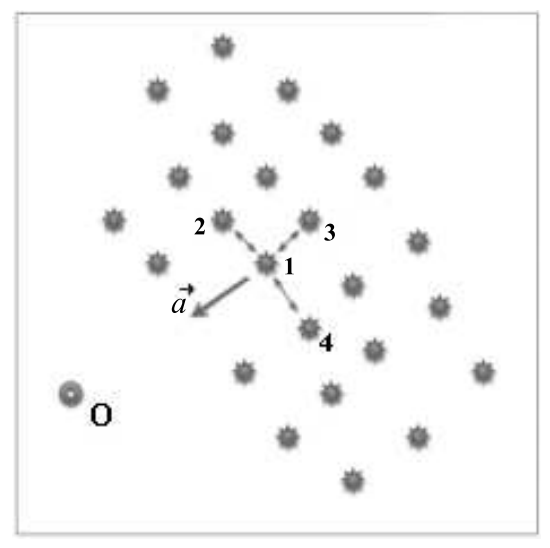

Figure 2: Stars in a disc galaxy. The acceleration $\boldsymbol{a}$, which star 1 experiences to the centre $\mathrm{O}$ of the galaxy, competes with accelerations on the side of surrounding stars 2,3 , 4, etc., which restrain the motion of star 1 to the point $\mathrm{O}$.

$$
\begin{aligned}
\omega=\left(\frac{G M}{R^{2} g}\right) & \approx\left\{\frac{\left(6.67 \times 10^{-11}\right) \times\left(2 \times 10^{40}\right)}{\left(3.09 \times 10^{20}\right)^{2} \times\left(3.13 \times 10^{16}\right)}\right\}^{1 / 2} \\
& \simeq 2.1 \times 10^{-14}\left[\mathrm{~s}^{-1}\right] .
\end{aligned}
$$

Having known all the parameters, we may estimate the number of stars in a cluster (26): $\aleph \approx 1.5 \times 10^{4}$.

In this plane cluster each star is involved in three kinds of interactions whose energies are

$$
\begin{aligned}
& G M m / R \approx 8.63 \times 10^{41} \mathrm{~J}, \\
& G m^{2} / g \approx 8.52 \times 10^{37} \mathrm{~J}, \\
& \frac{1}{2} m \omega^{2} g^{2} \approx 4.32 \times 10^{36} \mathrm{~J} .
\end{aligned}
$$

Let us now evaluate the acceleration that each star experiences in the plane cluster:

$$
a=G M / R^{2} \approx 1.4 \times 10^{-11} \mathrm{~m} \mathrm{~s}^{-2}
$$

This value of the acceleration satisfies the conditions prescribed by [2, 3]: $a<<$ $a_{0} \simeq 1.21 \times 10^{-10} \mathrm{~m} \mathrm{~s}^{-2}$. Thus we do not need to assume an incomprehensible modernization of Newton's law, i.e. the substitution of the force $\mathbf{F}=m \mathbf{a}$ by a significantly less force $\mathbf{F}=m \cdot(\mathbf{a}-\Delta \mathbf{a})$ at $a<<a_{0}$. Stars are distributed by clusters and each star is strongly bounded with the other $\aleph-1$ cluster's stars. This bounding compensates the centripetal acceleration $a$, which directs stars to the centre of the gravitating potential of the total mass $M$ of the galaxy. Expression (31) demonstrates this balance of two competing forces. That is why a Keplerian law, $V \sim \sqrt{r}$, is substituted for the constant orbital velocity (11).

Let us discuss now the 3-D cluster solution (21). The observed cluster (the interacting cluster $1 \mathrm{E}$ 0657-558 [7]) has the total mass $M \sim 10^{14} \mathrm{M}_{\odot}$, the radius $R \approx$ $250 \mathrm{kpc}$ and the central mass density $\rho_{0}=3.85 \times 6 \mathrm{M}_{\odot} \mathrm{kpc}^{-3}$. Putting for the mass of a star $m=\mathrm{M}_{\odot}$, we obtain the mean distance between stars: $g=\left(\mathrm{M}_{\odot} / \rho_{0}\right)^{1 / 3}=$ $4.25 \times 10^{16} \mathrm{~m}$. Then the stability of the cluster in respect to its gravitational collapse is determined by the relationship (31): the gravitational attraction of stars to the 
centroid is retained by the elasticity of inerton waves in the cluster. The frequency of oscillating stars at their equilibrium positions owing to the overlapping of their inerton waves is

$$
\begin{aligned}
\omega=\left(\frac{G M}{R^{2} g}\right) & \approx\left\{\frac{\left(6.67 \times 10^{-11}\right) \times\left(10^{14} \times 2 \times 10^{32}\right)}{\left(7.71 \times 10^{21}\right)^{2} \times\left(4.25 \times 10^{16}\right)}\right\}^{1 / 2} \\
& \simeq 3.82 \times 10^{-13}\left[\mathrm{~s}^{-1}\right] .
\end{aligned}
$$

The number of stars in such cluster, as it follows from expression (21), $\aleph=$ $2.39 \times 10^{9}$.

In this 3-D spherical cluster each star participates in three kinds of interactions whose energies are

$$
\begin{aligned}
& G M m / R \approx 3.46 \times 10^{46} \mathrm{~J}, \\
& G m^{2} / g \approx 6.27 \times 10^{37} \mathrm{~J}, \\
& \frac{1}{2} m \omega^{2} g^{2} \approx 2.64 \times 10^{39} \mathrm{~J} .
\end{aligned}
$$

The acceleration to the centroid, which each star experiences in the spherical cluster, is

$$
a=G M / R^{2} \approx 2.24 \times 10^{-8} \mathrm{~ms}^{-2} .
$$

The acceleration (37) is opposite to the inequality $a<<a_{0}$ needed for the use of MOND [2]. Besides, the acceleration (37) is not compensated by the acceleration caused by the elastic interaction in the cluster of $\aleph$ stars: $\omega^{2} g \approx 6.2 \times 10^{-9} \mathrm{~m} \mathrm{~s}^{-2}$. These are the reasons why calculations [7] of the shear profile $\gamma(\theta)$ caused by a point mass, which included a correction based on the MOND approach, showed a discrepancy between the available mass and the too intensive X-rays. Clowe et al. [7] note that the dark matter in the cluster exceeds at least twice the baryonic mass component in a MOND regime. The same emphasizes McGauph [1].

However, the origin of so-called dark matter is nothing but the same stars, which are involved in the mutual interaction through their inerton waves. This means that data obtained from the observation of stars must be considered taking into account an inerton component bounding stars. An important role may play parameters in expressions (33) and (36).

Basic concepts of gravitational lensing [25, 26, 27] should also be modified perhaps a point mass approach with a correction based on MOND or the other model will require a substitution by an approach resting on the involvement of elastically interacting masses. In particular, it seems the deflection angle $\varphi=4 \mathrm{Gm} /\left(c^{2} r\right)$ of a point mass $m$, which includes the absolute value of the gravitational potential $G m / r$, can be modified as follows

$$
\tilde{\varphi}=\frac{4}{c^{2}}\left(\frac{G m}{r}+\frac{1}{2} \omega^{2} r^{2}\right) ;
$$

this is evident from the pair interactions of stars (15) and (16). In a cluster the second term in expression (38) tends to align the space deformed by the first term. This has to be typically for 2-D clusters (i.e. clusters in disc galaxies), which is apparent from expressions (33). In the case of 3-D clusters (rich clusters in galaxies) the second term may even prevail the first one, see expressions (36), namely, the 
second term prolongs the deflection angle $\varphi$ for larger distances at which the first Newton's term becomes already negligible.

The deflection angle (38) can be presented through a ratio of accelerations

$$
\tilde{\varphi}=\frac{4 G m}{c^{2} r}\left(1+\frac{a}{2 a_{\text {grav }}}\right)
$$

where $a=\omega^{2} r$ and $a_{\text {grav. }}=G m / r^{2}$, which brings the approach closer to the MOND hypothesis. In a general way, one has to take into account a sum of distributed point masses and the possible presence of an outside potential $-G M / R$.

Massey et al. [28] showed that dark matter does interact via gravity, which is most effectively probed through gravitational lensing. The correction to the deflection angle introduced in expression (38) discloses the reason for such behaviour of dark matter.

Maps of the large-scale distribution of dark matter, a network of filaments and their intersection revealed by Massey et al. [28] allow a reasonable interpretation in the framework of the present theory: standing inerton waves of large gravitating masses indeed must interfere quite similar to waves on the water surface. An evolution of such interference pattern tends to a peculiar gravitational background, or scaffold by Massey et al. [28], into which gas can accumulate, and stars can be built.

\section{Concluding remarks}

In the present work we have shown that the submicroscopic concept exhibits the gravitation as a dynamic phenomenon - no motion, no gravity, - and allows the derivation of the Newton's law of universal gravitation (10) starting from first submicroscopic principles of the constitution of real space [20]. Submicroscopic mechanics further introduces the correction (11) to this law. This correction makes it possible to derive exactly the same equations for the perihelion precession of Mercury, the light deflection by Sun and the gravitational redshift of light [21, which were derived by general relativity. Besides, the submicroscopic concept uncovers inner reasons for the Shapiro time delay (namely, the concept shows what exactly is hidden behind the fourth component of the Schwarzschild metric) [22]

The submicroscopic concept introduces the tessel-lattice of mother-space as a source and generator of matter and physics laws. The concept is fully deterministic, removes an action at-a-distance and introduces a short-range action, which is provided by photons in the area of electromagnetic phenomena and inertons in the areas of quantum physics and gravitation. The concept complete rejects dark things from the space and inputs an additional elastic interaction between gravitating objects caused by overlapping of object's inerton waves (the notion of dark energy can be reduced to structural peculiarities of the tessel-lattice at the universe scale, which requires a separate consideration).

Although Zwitcky [29] is treated as the "father" of dark matter concept, the physical solution to this problem was demonstrated by Poincaré [30] even three decades before: describing the motion of an electron in the ether Poincaré noted that the electron was surrounded by excitations of the ether. Those excitations 
are interpreted as inertons of the submicroscopic concept described in the present paper.

The submicroscopic concept allows us to launch a new project in astronomy, namely, the Inerton Astronomy in the nearest future.

\section{References}

[1] S. S. McGauph, A novel test of the modified Newtonian dynamics with gas rich galaxies, Phys. Rev. Lett. 106:121303 (2011); arXiv: 1102.3913 [astro-ph.CO].

[2] A. Milgrom, A modification of the Newtonian dynamics as a possible alternative to the hidden mass hypothesis, Astrophys. J. 270, 365-370 (1983).

[3] A. Milgrom, The MOND limit from space-time scale invariance, Astrophys. J. 698, 1630-1639 (2009); arXiv: 0810.4065 [astro-phys.].

[4] R. H. Sanders, Modified Newtonian dynamics: a falsification of cold dark matter, Advances in Astronomy, vol. 2009, Article ID 752439, 9 pages, 2009. doi:10.1155/2009/752439.

[5] K. G. Begeman, A. H. Broeils, and R. H. Sanders, Extended rotation curves of spiral galaxies: dark haloes and modified dynamics, Mon. Not. R. Astron. Soc. 249, 523-537 (1991).

[6] R. H. Sanders, Clusters of galaxies with modified Newtonian dynamics, Mon. Not R. Astron. Soc. 342, No. 3, 901-908 (2003).

[7] D. Clowe, A. Gonzalez and M. Markevich, Weak-lensing mass reconstruction of the interacting cluster 1E 0657-558: Direct evidence for the existence of dark matter, Astrophys. J. 604, 596-603 (2004).

[8] L. de Broglie, Interpretation of quantum mechanics by the double solution theory, Ann. Fond. L. de Broglie 12, No. 4, 399-421 (1987).

[9] M. Bounias and V. Krasnoholovets, Scanning the structure of ill-known spaces: Part 1. Founding principles about mathematical constitution of space, Kybernetes: The Int. J. Systems and Cybernetics 32, No. 7/8, 945-975 (2003); arXiv: physics/0211096.

[10] M. Bounias and V. Krasnoholovets, Scanning the structure of ill-known spaces: Part 2. Principles of construction of physical space, ibid. 32, No. 7/8, 976-1004 (2003); arXiv: physics/0212004.

[11] M. Bounias and V. Krasnoholovets, Scanning the structure of ill-known spaces: Part 3. Distribution of topological structures at elementary and cosmic scales, ibid. 32, No. 7/8, 1005-1020 (2003); arXiv: physics/0301049.

[12] M. Bounias and V. Krasnoholovets, The universe from nothing: A mathematical lattice of empty sets, Int. J. Anticipatory Computing Systems 16, pp. 3-24 (2004), Ed.: D. Dubois; arXiv: physics/0309102.

[13] V. Krasnoholovets and D. Ivanovsky, Motion of a particle and the vacuum, Phys. Essays 6, No. 4, 554-563 (1993); arXiv: quant-ph/9910023.

[14] V. Krasnoholovets, Motion of a relativistic particle and the vacuum, Phys. Essays 10, No. 3, 407-416 (1997); arXiv: quant-ph/9903077. 
[15] L. de Broglie, Les insertitudes d'Hesenberg et l'interprétation probabiliste de la méchanique ondulatoire (Gausier-Villars, Bordas, Paris, 1982), Ch. 1.

[16] V. Krasnoholovets, Inerton fields: Very new ideas on fundamental physics, American Inst. Phys. Conf. Proc. - Volume 1316, (2010), pp. 244-268. Search for fundamental theory: The VII international symposium honoring French mathematical physicist Jean-Pierre Vigier (12-14 July 2010, Imperial College, London); doi:10.1063/1.3536437.

[17] V. Krasnoholovets, J.-L. Tane, An extended interpretation of the thermodynamic theory including an additional energy associated with a decrease in mass, Int. J. Simulation and Process Modelling 2, Nos. 1/2, 67-79 (2006); arXiv: physics/0605094.

[18] V. Krasnoholovets, Variation in mass of entities in condensed media, Appl. Phys. Research 2, No. 1, 46-59 (2010); http://www.ccsenet.org/journal/index.php/ apr/article/view/4287/4842.

[19] V. Krasnoholovets, N. Kukhtarev, T. Kukhtareva, Heavy electrons: electron droplets generated by photogalvanic and pyroelectric effects, Int. J. Modern Phys. B 20, No. 16, 2323-2337 (2006); arXiv: 0911.2361[quant-ph].

[20] V. Krasnoholovets, Reasons for the gravitational mass and the problem of quantum gravity, in Ether spacetime and cosmology, Vol. 1, Eds.: M. Duffy, J. Levy and V. Krasnoholovets (PD Publications, Liverpool, 2008), pp. 419-450 ( ISBN 187369410 5); arXiv.org: 1104.5270 (physics.gen-ph).

[21] V. Krasnoholovets, On microscopic interpretation of phenomena predicted by the formalism of general relativity, in Ether space-time and cosmology, Vol. 2: New insights into a key physical medium. Eds.: M. C. Duffy, J. Levy (Apeiron, 2009), pp. 417-431. (Publisher: C. Roy Keys Inc. Apeiron, ISBN: 0973291184; 978-0973291186); also: Apeiron 16, No. 3, 418-438, http://redshift.vif.com/ JournalFiles/V16NO3PDF/V16N3KRA.pdf.

[22] V. Krasnoholovets, On the gravitational time delay effect and the curvature of space, submitted, accepted.

[23] V. Krasnoholovets, B. Lev, Systems of particles with interaction and the cluster formation in condensed matter, Cond. Matt. Phys. 6, No. 1, 67-83 (2003); arXiv: cond-mat/0210131.

[24] B. Fort, Y. Mellier, Arc(let)s in clusters of galaxies, The Astronomy and Astrophysics Review 5, 239-292 (1994).

[25] P. V.Bliokh, A. A. Minakov, Gravitational Lenses (Naukova Dumka, Kyiv, 1989) (in Russian)

[26] P. Schneider, J. Ehlers, E. E. Falco, Gravitational Lenses (Springer-Verlag, Berlin, 1992).

[27] D. J. Mortlock, E. L. Turner, Gravitational lensing in modified Newtonian dynamicsm, Mon. Not. R. Astron. Soc. 327, 557-566 (2001).

[28] R. Massey, J. Rhodes, R. Ellis, N. Scoville, A. Leauthaud, Finoguenov, P. Capak, D. Bacon, H. Aussel, J.-P. Kneib, A. Koekemoer, H. McCracken, B. 
Mobasher, S. Pires, A. Refregier, S. Sasaki, J.-L. Starck, Y. Taniguch, A. Taylor, J. Taylor, Dark matter maps reveal cosmic scaffolding, Nature 445, 286-290 (2007); doi:10.1038/nature05497.

[29] F. Zwicky, Die Rotverschiebung von extragalaktischen Nebeln, Helvetica Physica Acta 6: 110-127 (1993).

[30] H. Poincaré, Sur la dynamique de l'électron, Comptes Rendus 140, 15041560, (1905); textitRendiconti del Circolo matematico di Palermo 21, 129-176 (2006); also: Oeuvres, t. IX, pp. 494-550 (and also in Russian translation: Selected Transactions, ed. N. N. Bogolubov (Nauka, Moscow, 1974), 3, 429486). 